\title{
A Game Theoretic Solution for Exploiting Multiuser Diversity in Cooperative Slotted Aloha
}

\author{
Cristina Comaniciu ${ }^{\dagger}$, Dandan Wang*, Hlaing Minn* and Naofal Al-Dhahir* \\ ${ }^{\dagger}$ Dept. of Electrical and Engineering, Stevens Institute of Technology, \\ Hoboken, NJ 07094, USA. Email: \{ccomanic\}@ stevens.edu \\ ${ }^{*}$ Dept. of Electrical and Engineering, University of Texas at Dallas, Richardson, Tx, 75083, USA. \\ Emails:\{dxw053000, hlaing.minn, aldhahir\}@utdallas.edu
}

\begin{abstract}
1
Abstract - In multiuser systems, multiuser diversity gains can be achieved by assigning channels to users with higher channel quality. To avoid the extensive information exchange required for centralized approaches, we propose a distributed fair pricing strategy for a slotted Aloha system in which users act selfishly to improve their own utilities. Based on a game theoretic framework, we show that multiuser diversity gains can be achieved by appropriately designing the Nash equilibrium thresholds for the selfish users, such as to preserve the throughput and revenue achieved in the centralized schemes. The network enforces fairness among different users by employing a pricing policy that favors equal access probabilities. Our simulation results show significant multiuser diversity gains achieved in terms of energy consumption and/or spectral efficiency.
\end{abstract}

\section{INTRODUCTION}

A fundamental characteristic of wireless mobile communication systems is random channel fading. Traditionally, to combat the detrimental effects of channel fading, diversity in time, space and frequency domains can be exploited. Instead of mitigating unreliably fading for each user, multiuser diversity has been proposed to maximize the total informationtheoretic capacity in the context of multiuser communications [1][2]. The basic idea of multiuser diversity is to exploit the randomness of the fading channels among different users. The larger the dynamic range of the channel fluctuations and the number of users, the larger the multiuser diversity gain. In a centralized system, the access point assigns the channel to the user with the best instantaneous channel as in [1],[3]. However, centralized scheduling schemes require significant information exchange between users and the access point. Consequently, a distributed access scheme which can reduce this information exchange while exploiting the multiuser diversity will be highly desirable. In [4], a distributed channel aware slotted Aloha is proposed in which each user only utilizes his own channel information and assumes that users will implement the same mandated algorithm and behave in a predictable manner which is set by the network. However, we note that

\footnotetext{
${ }^{1 \dagger}$ This work was supported in part by NSF grant number CNS-0435297, and by iNetS (Center for Intelligent Networked Systems).

*The work of this author was supported in parts by the Erik Jonsson School Research Excellence Initiative, the University of Texas at Dallas, USA, and the National Science Foundation (NSF) under Contracts CCF 04-30654 and DMS 05-28010.
}

in a distributed system, users may act selfishly to maximize their own performance.

In this paper, we use a game theoretic approach to analyze selfish users' behavior in a distributed multiuser diversity scheme, and to regulate the overall network performance via enforcing a fair pricing strategy. Fairness imposes the constraint of an equal access probability. This pricing based design incentives all selfish users to cooperate with each other. We design the pricing based medium access control (MAC) algorithm such as to obtain diversity gains, while preserving the optimum throughput and revenue that can be achieved in a centralized network.

Game theoretic formulations for analyzing MAC protocols in the presence of selfish users that seek to maximize their own performance $([5][9][10][11][6][7])$, in particular for slotted Aloha, were recently proposed in the literature, including the work in [8][13][12]. In our previous work in [13][12], we show that by enforcing cooperation through pricing mechanisms, the throughput of the centralized slotted Aloha can be achieved in a distributed network in which selfish users access the network attempting to maximize their own utility. However, our proposed MAC schemes in [13] and [12] do not achieve multiuser diversity gains. In fact, to the best of our knowledge, none of the previously proposed game theoretic formulations for MAC algorithms have considered multiuser diversity as a tool to enhance the performance. This motivates our research presented here. In this paper, we propose a new game model which associates the channel characteristics with the transmission costs to achieve multiuser diversity gains. As opposed to our previous approach in [13], where we have determined a Nash equilibrium transmission probability, here we design a Nash equilibrium threshold that accounts for statistical channel characteristics. Each user decides whether to transmit or wait by comparing his transmission cost with the Nash threshold. As opposed to [13], the network charges the same price for all successful packets, while the pricing varied with the channel characteristic in [13]. We consider two different optimization criteria for our MAC algorithm: throughput and revenue maximization, and we impose an equal access probability solution to enforce fairness. Both theoretical and simulation results show that the pricing model proposed in this paper leads to a distributed MAC algorithm that consumes less energy and achieves a higher spectral efficiency. 
The paper is organized as follows. In Section II, a game theoretic formulation for exploiting multiuser diversity is proposed. The analysis of the Nash equilibrium for a collision model channel is presented in Section III. Simulation results are shown in Section IV. Conclusions are presented in Section $\mathrm{V}$.

\section{GAME MODEL FOR EXPLOITING MULTIUSER DIVERSITY GAINS}

In this section, we present a game-theoretic model for a distributed slotted system. We assume that all users can estimate their own channel characteristics by listening to a periodically-transmitted beacon signal from the base station (see also [4]).

Each slot of the system is a one-stage game. At the beginning of each slot, all users learn the current state of the game, which is $N$, the number of users who currently have packets to send (active users). We note that, in practical systems, the number of active users can also be estimated if a pseudo-Bayesian Aloha algorithm is employed [14]. In this paper, we investigate the scenario where all users always have data to transmit (high load systems), i.e. all the users are active users. Each of these users has two possible actions: transmit (T) or wait (W). When a user transmits, its transmission can either succeed (S) or fail (F). The gain associated with a successful transmission is a normalized throughput of 1 , while the cost of transmission for user $i$ is $c_{i}$, and the network's current charge for each successful packet is $\mu$, which is the same for all users. If user $i$ transmits and succeeds in a given slot, then that user will receive a payoff of $1-c_{i}-\mu$ for that slot (throughput - cost - price paid). If the user refrains from transmission in a particular slot (waits), this will result in one slot delay for that user. This delay is associated with the loss in the throughput the user could have achieved if it transmitted and succeeded. Thus, the payoff for this waiting user $i$ can be determined as $-\left(1-c_{i}-\mu\right)$ (the negative of the payoff it could have been achieved if successful). Note that this cost model assumes that users use the system resources aggressively. They value an opportunity to send, such that they believe that if they would have sent, they would have been successful in their transmission. For that reason, the waiting cost for these users is only associated with the payoff in case the users successfully access the network.

If user $i$ transmits but fails, it will incur a transmission $\operatorname{cost} c_{i}$ as well as one slot delay, but it will not pay for the transmission (we assume that a user is charged only for successful transmissions). Therefore, the payoff of this user in this case can be defined as $-\left(1-c_{i}-\mu\right)-c_{i}=-1+\mu$. Each user's goal is to maximize its own payoff. The payoff function for user $i$ is summarized in Table I.

\begin{tabular}{|c|c|c|}
\hline T and $\mathrm{S}$ & $\mathrm{T}$ but $\mathrm{F}$ & $\mathrm{W}$ \\
\hline $1-c_{i}-\mu$ & $-1+\mu$ & $-\left(1-c_{i}-\mu\right)$ \\
\hline
\end{tabular}

TABLE I
Please note that in our previously proposed pricing model in [13], the network charges differently for different users. When a user's transmission cost is high, the network charges less to encourage that user to transmit, such that fairness can be achieved.

In this paper, our optimization goal is different: the network charges the same price for all users, such that the users with lower transmission costs are encouraged to transmit. Fairness in this case can be guaranteed by properly designing an equilibrium threshold associated with this uniform pricing.

It is important to notice that the transmission cost $c_{i}$ should be a normalized function in the range of $(0,1)$ and is related to the energy cost for transmission. In wireless systems, channels change from one slot to another slot. The higher the channel power gain, the less the transmission power (hence transmission cost) to achieve the same spectral efficiency. Thus, the transmission cost should be defined as a monotonic decreasing function of channel power gains. Let $G_{i}$ denote the channel power gain of the $i$-th user and $F_{i}($.$) denote$ the cumulative probability distribution function (CDF) of $G_{i}$. We define the transmission cost to be $c_{i}=1-F_{i}\left(G_{i}\right)$. This definition preserves the required properties for $c_{i}$, i.e., normalization, and decreasing monotonicity with the increase of the channel power gains. Please note that there may be some other definition of $c_{i}$ which satisfies the required properties. Our definition simplify the theoretical analysis and also has practical interests.

Our goal is to optimize the system performance by appropriately selecting the price $\mu$, according to users' transmission costs $c_{j}, j=1,, \cdots, N$, so that each user can achieve its maximum payoff by taking appropriate actions: $\mathrm{T}$ or $\mathrm{W}$.

\section{EQUILIBRIA FOR THE COLLISION MODEL}

In this section, we consider the classical collision model, for which a transmission is considered to be successful only when a single user transmits.

In the game model described in the previous section, every user has two strategies, transmit (T) or wait (W). If the payoff of $\mathrm{T}$ is larger than $\mathrm{W}$, then the user transmits. Otherwise, he waits. An equilibrium strategy will be a vector of actions ( $T$ or $W$ ) each user takes in this slot: $A=(T / W, T / W, T / W$, ...), such that $A$ maximizes each user's payoff given that there are currently $N$ users in the game. The equilibrium changes from one slot to another as the transmission costs change.

In this paper, we consider a fair game model, i.e. all users access the system with the same probability $p$. Then the payoff user $i$ gets from selecting action $\mathrm{T}$ is

$$
\begin{aligned}
v(T, i) & =(-1+\mu)\left[1-(1-p)^{N-1}\right] \\
& +\left(1-c_{i}-\mu\right)(1-p)^{N-1} .
\end{aligned}
$$

The payoff user $i$ gets with action $\mathrm{W}$ is

$$
v(W, i)=-\left(1-c_{i}-\mu\right)
$$


In [13], a nondegenerate mixed strategy equilibrium from (1) and (2) is obtained using the indifference principle. However, the mixed strategy equilibrium can not achieve multiuser diversity gains. In this paper, we obtain multiuser diversity gains by finding an equilibrium threshold. To maximize its own payoff, a user will take an action of $T$ when $v(T, i)>$ $v(W, i)$. Otherwise, it will wait. From (1) and (2), we can obtain

$$
v(T, i)>v(W, i) \Rightarrow c_{i}<\frac{2(1-\mu)(1-p)^{N-1}}{1+(1-p)^{N-1}} .
$$

Define

$$
T_{h}=\frac{2(1-\mu)(1-p)^{N-1}}{1+(1-p)^{N-1}},
$$

where $T_{h}$ is called the equilibrium threshold and we can obtain

$$
c_{i}<T_{h}
$$

Then the problem of finding a Nash equilibrium strategy is reduced to the one of finding an equilibrium threshold. The users whose transmission costs are smaller than the equilibrium threshold will transmit their packet at this time slot. Since we only consider the classical collision channel model in this paper, only if there is exactly one user whose transmission cost is smaller than the equilibrium threshold, the transmission is successful.

Since the successful user is the only one attempting transmission, according to our Nash equilibrium threshold design, it must be the user with the lowest transmission cost and consequently the highest channel gain. In this way, our game model achieves multiuser diversity gains.

Now, let us analyze the relationship among $T_{h}, p$ and $\mu$. As $p$ is the probability that a user transmits, we have

$$
p=P\{v(T, i)>v(W, i)\}=P\left\{c_{i}<T_{h}\right\} .
$$

Substituting $c_{i}=1-F_{i}\left(G_{i}\right)$ into (6), we obtain

$$
P\left\{G_{i}>F_{i}^{-1}\left(1-T_{h}\right)\right\}=p .
$$

Thus,

$$
1-F_{i}\left\{F_{i}^{-1}\left(1-T_{h}\right)\right\}=p .
$$

Further simplifying (8), we obtain

$$
T_{h}=p
$$

Substituting (8) into (4) gives

$$
\mu=1-\frac{p+p(1-p)^{N-1}}{2(1-p)^{N-1}} .
$$

It is interesting to note that the equilibrium threshold is the same as the transmission probability $p$, which is decided by the price charged by the network, $\mu$. To obtain a larger $p$, the network needs to reduce the pricing. In turn, the transmission probability will influence the achievable network throughput, and in combination with the pricing scheme, the revenue of the network provider.

Consequently, we can optimize the pricing scheme, given the two above mentioned criteria: throughput and revenue.

\section{A. Throughput Optimization}

This subsection describes how the network adjusts pricing to achieve the maximum throughput. As we know, the network throughput $S$ of a centrally controlled system is

$$
S=N p(1-p)^{N-1},
$$

and the maximum throughput is achieved when $p=1 / N$ [14]. Therefore, from (10), the optimal pricing to maximize the throughput is,

$$
\mu=1-\frac{\frac{1}{N}+\frac{1}{N}\left(1-\frac{1}{N}\right)^{N-1}}{2\left(1-\frac{1}{N}\right)^{N-1}} .
$$

The equilibrium threshold is

$$
T_{h}=p=\frac{1}{N}
$$

At the first glance, it may seem that pricing does not have any effect on the performance since the equilibrium threshold is equal to $\frac{1}{N}$. However, without pricing, i.e. $\mu=0$, we have

$$
p=T_{h}=\frac{2(1-p)^{N-1}}{1+(1-p)^{N-1}} .
$$

The numerical solutions for (14), for different number of users, are given in Fig. 1, and it can be seen that it is different from our proposed pricing solution which maximizes the throughput (for $T_{h}=\frac{1}{N}$ ). Thus, by introducing pricing, the network can regulate the overall network behavior.

Pricing $\mu$ is monotonically increasing with the number of users as shown in Fig.2 (the curve denoted as 'Throughput optimization'). This result is intuitively appealing, since the network should reduce its charging price to encourage users to transmit when there are only few users in the system. When $N=1$, there is only one user in the system. In this case, $\mu=0$ and $T_{h}=1$ so that this user is always transmitting data. For the extreme case of $N=\infty, \mu=1$ and $T_{h}=0$ and no user will try to transmit since there will be always collisions.

\section{B. Revenue Optimization}

In the previous subsection, the network decides the price aiming to maximize the throughput of the whole network. In this subsection, we consider a more realistic objective for the service provider which is to maximize revenue. Assume that the network charges only for successful transmissions. To achieve fairness, the transmission probabilities for all users are enforced to be the same and hence the revenue for the network of a centrally controlled system is given by

$$
R=\mu S=\mu N p(1-p)^{N-1}
$$

Substituting $\mu$ given in (10) into (15), we obtain

$$
R=\left(1-\frac{p+p(1-p)^{N-1}}{2(1-p)^{N-1}}\right) N p(1-p)^{N-1} .
$$


The optimum $p$ can be obtained by setting the derivative of (16) to zero. The closed form solution is analytically intractable. However, several simulation tools can be used to compute the numerical solutions, such as the fminsearch function in MATLAB. After we obtain the optimum solution, the price $\mu$ can be obtained from (10) and the equilibrium threshold can be obtained from (9).

Now we will investigate the relationship between these two criteria. It is well-known from the centrally-controlled slotted Aloha protocol that $S=N p(1-p)^{N-1}$ is a strictly-increasing function over $p$ when $p<\frac{1}{N}$, and a strictly-decreasing function when $p>\frac{1}{N}$ [14]. The maximum of $S$ is $\frac{1}{e}$ achieved at $p=\frac{1}{N}$.

From (10), it can be seen that $\mu$ is a decreasing function of $p$. Therefore, the optimum $p$ maximizing $R$ must be no larger than the optimum $p$ maximizing $S$. Then, the optimum pricing for the revenue optimization should be no smaller than the optimum pricing for the throughput optimization. We can also see these facts from Fig. 1 and Fig. 2. Similar to the case of the throughput optimization, the pricing for the revenue optimization also increases with the number of users. The pricing for the revenue optimization is slightly larger than that for the throughput optimization. Fig. 1 compares the Nash equilibrium thresholds for the two design criteria. The thresholds for the throughput optimization are slightly larger than the thresholds for the revenue optimization, such that, the transmission probability in the case of the throughput optimization is larger than the one obtained for the revenue optimization.

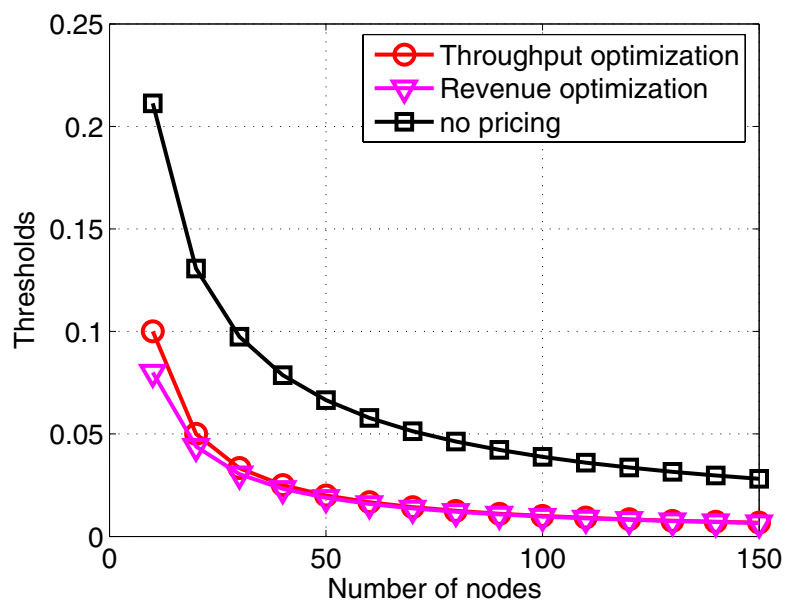

Fig. 1. Threshold comparison

\section{Numerical and Simulation Results}

In the previous sections, we have described the game model for our MAC access scheme and we have derived the Nash equilibrium thresholds for it. In this section, both simulation results and numerical results are provided. The simulation results are obtained from 100,000 independent frames and we

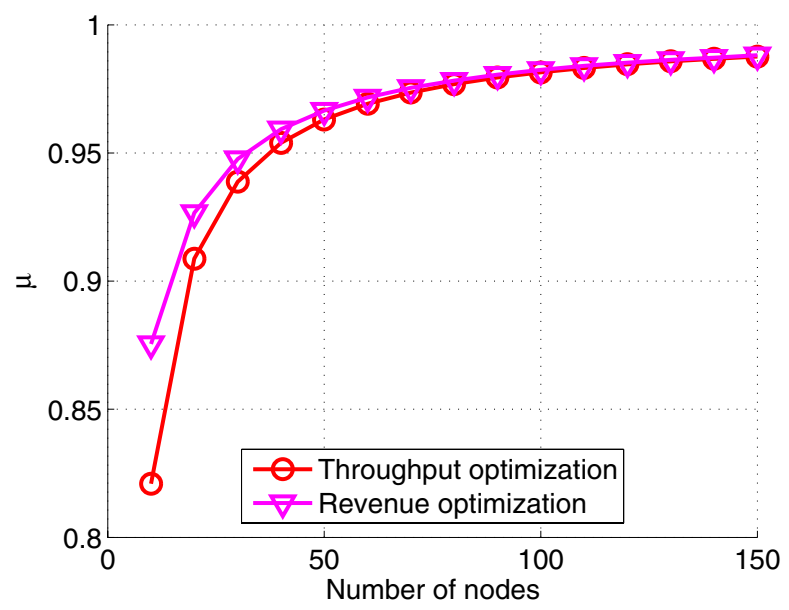

Fig. 2. Pricing comparison

assume a Bit-error-Rate requirement of BER $=10^{-5}$ and a corresponding Signal-to-noise ratio requirement of $S N R=15 \mathrm{~dB}$. To show the multiuser diversity gains in the simulation, we will consider two performance criteria: energy consumption and spectral efficiency. They are defined as follows.

1) Spectral efficiency The spectral efficiency metric is used to measure how many bits a user can transmit for a given bandwidth. Here, a continuous rate adaptive modulation is used by exploiting the instantaneous channel power gains, and the spectral efficiency is calculated as

$$
r=\log _{2}\left(1+\frac{\gamma P G_{i}}{\sigma_{i}^{2}}\right),
$$

where $P$ is the transmission power, $\sigma_{i}^{2}$ is the noise power and $\gamma$ is the SNR gap given by

$$
\gamma=-\frac{1.5}{\ln (5 \mathrm{BER})}
$$

Note that to show the effects of the channel gains on the number of bits transmitted, $P$ is considered fixed for all the users and is set to be ' 1 ' in the simulation.

2) Energy consumption The energy consumption metric is used to evaluate how much energy is consumed by various users to achieve a given data rate, given the same pre-specified BER requirement.

Note that the users who transmit but fail, will still consume the same amount of energy as if they transmitted and succeeded as defined in the game model in Section II. The difference is that they do not need to pay anything if their packets are not successfully received. From (17), we can calculate the power $P$ to achieve data rate $r$ as

$$
P=\frac{\left(2^{r}-1\right) \sigma_{i}^{2}}{\gamma G_{i}}
$$


In this simulation, we use the normalized data rate $r=$ 1 , and $\gamma$ is defined as in (18). It can be seen from (19) that the higher the channel power gain of the user, the less its power consumption is.

To simplify the notations for illustration purposes, our proposed game scheme is denoted as COOP-MUD-ALOHA. We run simulations and present theoretical results to illustrate the performance of our new proposed scheme, and we compare the performance results with the following three benchmark algorithms:

- Conventional slotted Aloha (CONV-ALOHA) [14]: each user transmits with a probability $\frac{1}{N}$ irrespective of its channel characteristics.

- MAC scheme proposed in our previous work [13] (COOP-ALOHA).

COOP-ALOHA is a cooperative MAC scheme which enforces equal access probability for all the users. COOPALOHA does not achieve multiuser diversity gains. In [13], we gave an example of $c_{i}$ in terms of the fraction of the total battery energy level. For a fair comparison, in this paper, we redefine $c_{i}$ from [13] to be the same as the transmission cost in this paper, i.e., $c_{i}=1-F_{i}\left(G_{i}\right)$. This redefinition of $c_{i}$ does not affect the computed Nash equilibrium in [13].

- Non-optimum COOP-MUD-ALOHA: This scheme uses COOP-MUD-ALOHA with two randomly selected nonoptimum equilibrium thresholds- $T_{h}=\frac{1}{2 N}$ and $T_{h}=$ $\frac{2}{N}$, in order to show the effects of the equilibrium thresholds.

\section{A. System Model}

We assume that the channels between users and the access point are characterized by independent fading, and the received signal is Rayleigh distributed. The lowpass equivalent channel gains $\left\{h_{i},(i=1,2, \ldots N)\right\}$ between an arbitrary user $i$ and the access point are assumed to be independent zero mean unit variance circularly-symmetric complex Gaussian random variables. Consequently, the channel power gains $G_{i}=\left|h_{i}\right|^{2}$ $(i=1,2, \ldots, N)$ have exponential distribution with mean ' 1 ', i.e,

$$
F_{G}(g)=\left(1-e^{-g}\right) u(g),
$$

where $u(g)$ is the unit step function. We obtain the transmission cost of user $i$ as

$$
c_{i}=1-F_{G}\left(G_{i}\right)=e^{-g} u(g) .
$$

\section{B. Numerical and Simulation Results}

Figs. 3, 4, 5 and 6 give the numerical and simulation results. From Fig. 3 and Fig. 4, we can see that our simulation results match very well with our theoretical results which are calculated from (11) and (15). COOP-MUD-ALOHA based on the throughput optimization shows almost the same performance as COOP-MUD-ALOHA based on the revenue optimization, and both are significantly better than the non-optimum COOPMUD-ALOHA scheme. It is interesting to note that both COOP-ALOHA and COOP-MUD-ALOHA have the same throughput performance, for the case of throughput optimization. However, if revenue is optimized, the revenue obtained for COOP-ALOHA is much less than that obtained by the COOP-MUD-ALOHA scheme.

The reason for the above observations is that in COOPALOHA, the price changes with the transmission costs, to encourage equal access probability even when the transmission cost is high, whereas in COOP-MUD-ALOHA the price remains the same, and it is usually higher than the one for COOP-ALOHA. In COOP-ALOHA, when the transmission cost of a user is larger, the network tends to reduce the price of that user to impose the equal access probability. By contrast, in COOP-MUD-ALOHA, it is always the user whose transmission cost is smallest that accesses the network. Therefore, most of the prices in COOP-MUD-ALOHA are higher than the prices in COOP-ALOHA.

Fig. 5 compares the spectral efficiencies of different schemes. COOP-MUD-ALOHA again achieves much higher spectral efficiency than other schemes for both the case of throughput optimization and revenue optimization.

Fig.6 gives the comparison of the energy consumption for different MAC schemes. It can be seen that COOPMUD-ALOHA consumes much less energy compared to both CONV-ALOHA and COOP-ALOHA. It is worthy to note the impact of the equilibrium threshold selection. Non-optimum COOP-MUD-ALOHA with $T=\frac{1}{2 N}$ saves more energy than COOP-MUD-ALOHA, as a smaller threshold reduces the transmission probability for all users. However, its throughput, revenue and spectral efficiency are less than the optimum COOP-MUD-ALOHA, as shown in Figs. 3, 4 and 5.

From the presented simulation results, we can see that our proposed COOP-MUD-ALOHA achieves much higher multiuser diversity gains compared with the other schemes, without sacrificing throughput or revenue. In addition, throughput optimization based COOP-MUD-ALOHA has almost the same performance as the revenue optimization based COOP-MUDALOHA. However, for COOP-ALOHA in [13], the network must tradeoff throughput optimization and revenue optimization as shown in Figs. 3 and 4.

Finally, we should point out that although the simulation assumes that all the users have the same channel statistic, COOP-MUD-ALOHA will also guarantee fairness (due to enforced equal access probability design), even for the case of a heterogeneous system, which is characterized by having different channel statistics for various users.

\section{CONClusion}

In this paper, we have studied the problem of enforcing cooperation and fairness for selfish users in slotted Aloha to achieve multiuser diversity gains. Energy and spectral efficiency, as well as network throughput and revenue for the service provider were considered as performance metrics. Our analysis is based on a game theoretic framework. We have showed that, if the users' payoff is modified to account for network pricing, multiuser diversity gain can be achieved without sacrificing throughput or revenue. These multiuser 


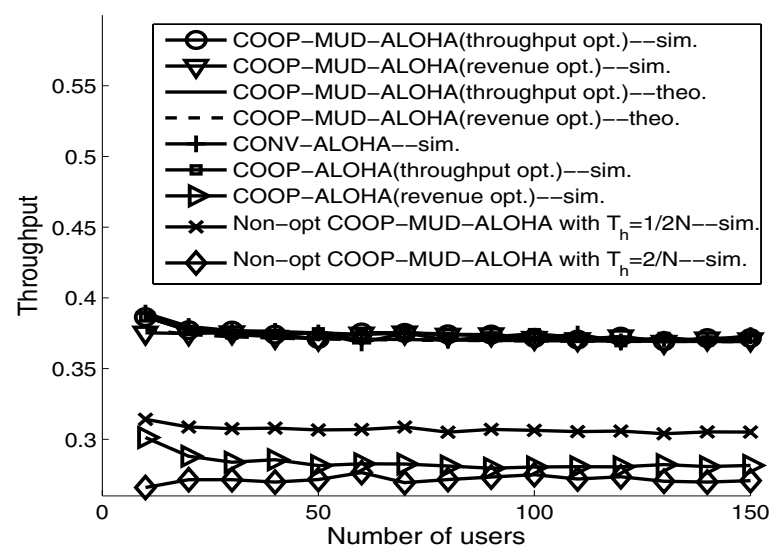

Fig. 3. Throughput comparison

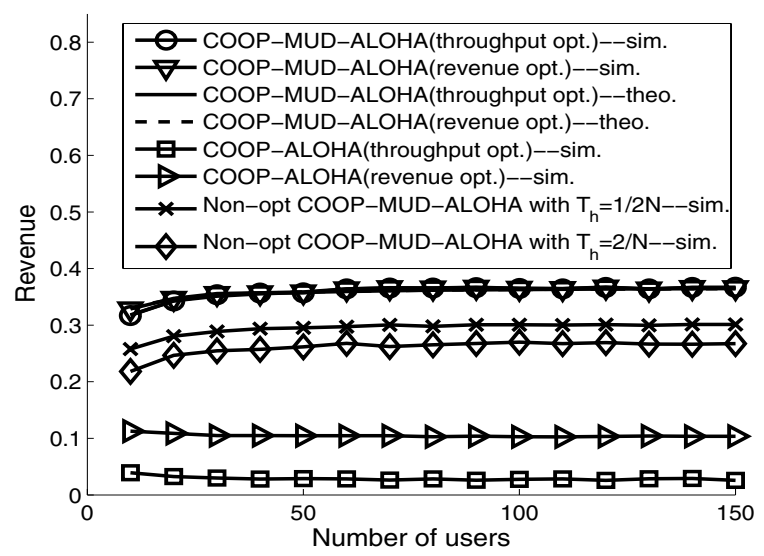

Fig. 4. Revenue comparison

diversity gains make the systems more energy efficient and can offer higher spectral efficiency. Our results prove the viability of distributed MAC implementation in networks with selfish users, and determine the required policy to enforce cooperation and fairness in such networks, in order to optimize the overall network performance.

\section{REFERENCES}

[1] R. Knopp and P. A. Humblet, Information capacity and power control in single-cell multiuser communications, Proc. of Intl. Conf. on Com., Seattle, WA, pp. 331-335, June 1995.

[2] D. N. C. Tse, Optimal power allocation over parallel Gaussian channels, Proc. Int. Symp. Information Theory, Ulm, Germany, pp.27-27, June 1997.

[3] P. Viswanath, D. N. C. Tse and R. Laroia, Opportunistic beamforming using dumb antennas, IEEE Trans. Inform. Theory, Vol. 48, No. 6, pp. 1277-1294, June 2002.

[4] X. Qin and R. Berry, Exploiting multiuser diversity for medium access in wireless networks, Proc. of INFOCOM Conf., San Francisco, CA, vol. 2, pp. 1084-1094, Mar. 30-April 3, 2003.

[5] Nan Feng, Siun-Chuon Mau, Narayan N.B, pricing and power control for joint network-centric and user-centric radio resource management, IEEE Trans. Communications, Pages:1547-1557, vol. 52, no. 9, Nov,2004.

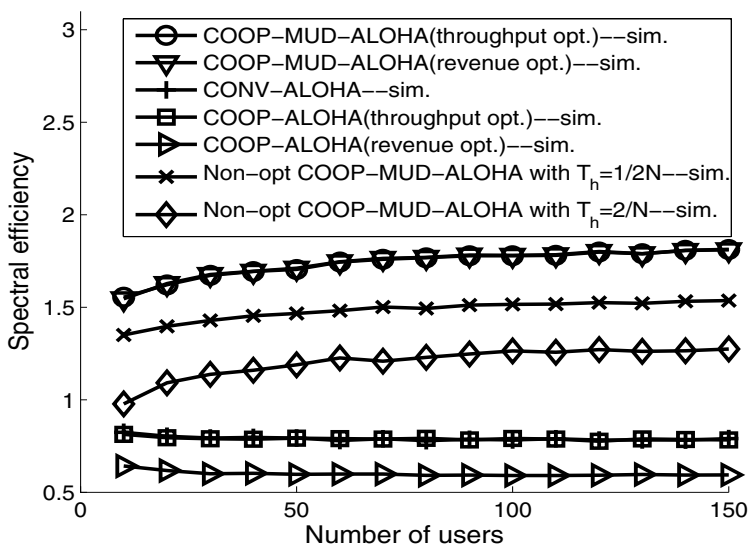

Fig. 5. Spectral efficiency comparison

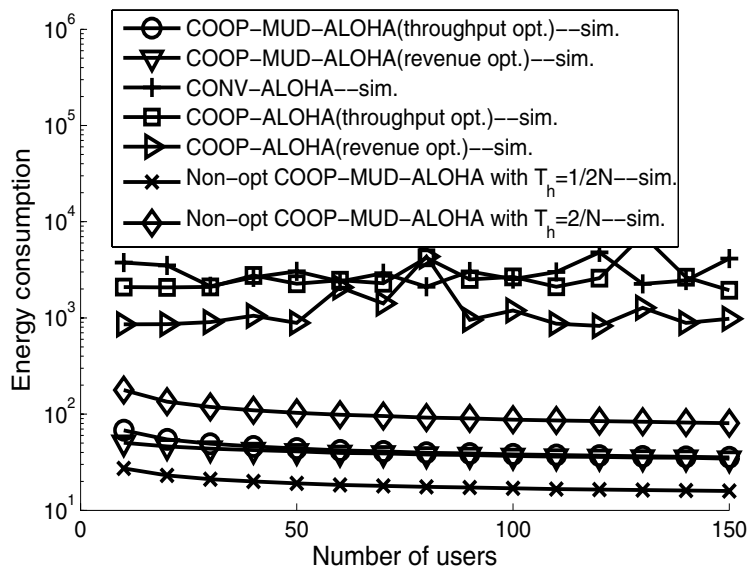

Fig. 6. Energy consumption comparison

[6] R.D. Yates, A framework for uplink power control in cellular radio systems, IEEE JASC, Pages:1341-1347, vol. 13, Issue 7, Sept., 1995.

[7] E. Baccarelli, M. Biagi, C. Pelizzoni, A distributed power-allocation and signal-shaping game for the competitively optimal throughputmaximization of multiple-antenna "ad hoc" networks, IEEE Trans. Veh. Tech., Pages:1862-1876, vol. 55, Issue 9, Nov,2004.

[8] A. B. MacKenzie and S. B. Wicker, Selfish users in Aloha: A gametheoretic approach, IEEE Vehicular Technology Conference (VTC) Fall 2001, vol.3, pp:1354-1357, Atlantic City, NJ, Oct. 2001.

[9] E. Altman, D. Barman, R. El Azouzi, and T. Jimenez, A game theoretic approach for delay minimization in slotted Aloha, IEEE International Conference on Communications (ICC) 2004, vol. 7, pp: 3999-4003, Paris, 20-24 June 2004.

[10] Y.Jin and G.Kesidis, A pricing strategy for an Aloha network of heterogeneous users with inelastic bandwidth requirements, In Proceeding of 39th Annual Conference on Information Sciences and Systems, 2002.

[11] J. Sun and E. Modiano, Opportunistic Power Allocation for Fading Channels with Non-cooperative Users and Random Access, IEEE BroadNets C Wireless Networking Symposium, October, 2005.

[12] D. Wang, C. Comaniciu and U. Tureli, A fair and efficient pricing strategy for slotted Aloha in MPR models, IEEE VTC, September 2006

[13] D. Wang, C. Comaniciu and U. Tureli, Cooperation and Fairness for Slotted Aloha, to appear in Special Issue on Cooperation in WirelessNetworks, Springer-Wireless Personal Communication.

[14] D. Bertsekas and R. Gallager, Data Networks, 2nd edition (Prentice-Hall, Upper Saddle River, NJ, 1992). 